\title{
Identifying Opioid Use Disorder in the Emergency Department: Multi-System Electronic Health Record-Based Computable Phenotype Derivation and Validation Study
}

David Chartash ${ }^{1}$, PhD; Hyung Paek ${ }^{2}$, MSEE, MD; James D Dziura ${ }^{3}$, MPH, PhD; Bill K Ross ${ }^{4}$; Daniel P Nogee ${ }^{3}$, MD; Eric Boccio ${ }^{3}$, MD; Cory Hines ${ }^{5}$, MD; Aaron M Schott ${ }^{5}$, MD; Molly M Jeffery ${ }^{6,7}$, PhD; Mehul D Patel ${ }^{5}$, PhD; Timothy F Platts-Mills ${ }^{5}$, MSc, MD; Osama Ahmed ${ }^{3}$, BSc; Cynthia Brandt ${ }^{1,3}$, MPH, MD; Katherine Couturier ${ }^{3}$, MD; Edward Melnick ${ }^{3}$, MHS, MD

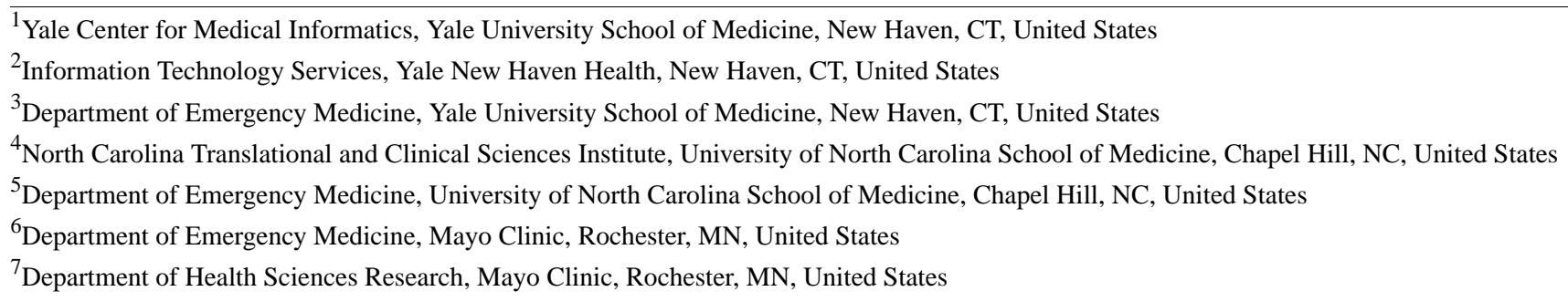

Corresponding Author:

Edward Melnick, MHS, MD

Department of Emergency Medicine

Yale University School of Medicine

464 Congress Ave

Suite 260

New Haven, CT, 06519

United States

Phone: 12037855174

Email: edward.melnick@yale.edu

\section{Abstract}

Background: Deploying accurate computable phenotypes in pragmatic trials requires a trade-off between precise and clinically sensical variable selection. In particular, evaluating the medical encounter to assess a pattern leading to clinically significant impairment or distress indicative of disease is a difficult modeling challenge for the emergency department.

Objective: This study aimed to derive and validate an electronic health record-based computable phenotype to identify emergency department patients with opioid use disorder using physician chart review as a reference standard.

Methods: A two-algorithm computable phenotype was developed and evaluated using structured clinical data across 13 emergency departments in two large health care systems. Algorithm 1 combined clinician and billing codes. Algorithm 2 used chief complaint structured data suggestive of opioid use disorder. To evaluate the algorithms in both internal and external validation phases, two emergency medicine physicians, with a third acting as adjudicator, reviewed a pragmatic sample of 231 charts: 125 internal validation (75 positive and 50 negative), 106 external validation (56 positive and 50 negative).

Results: Cohen kappa, measuring agreement between reviewers, for the internal and external validation cohorts was 0.95 and 0.93, respectively. In the internal validation phase, Algorithm 1 had a positive predictive value (PPV) of 0.96 (95\% CI 0.863-0.995) and a negative predictive value (NPV) of 0.98 (95\% CI 0.893-0.999), and Algorithm 2 had a PPV of 0.8 (95\% CI 0.593-0.932) and an NPV of 1.0 (one-sided $97.5 \%$ CI $0.863-1$ ). In the external validation phase, the phenotype had a PPV of 0.95 (95\% CI 0.851-0.989) and an NPV of 0.92 (95\% CI 0.807-0.978).

Conclusions: This phenotype detected emergency department patients with opioid use disorder with high predictive values and reliability. Its algorithms were transportable across health care systems and have potential value for both clinical and research purposes. 
(JMIR Med Inform 2019;7(4):e15794) doi: 10.2196/15794

\section{KEYWORDS}

electronic health records; emergency medicine; algorithms; phenotype; opioid-related disorders

\section{Introduction}

\section{Background}

In the decade since the Health Information Technology for Economic and Clinical Health Act of 2009 was enacted, US hospitals have achieved greater than $96 \%$ adoption of electronic health records (EHRs) [1]. EHRs are projected to store 2314 exabytes $(1$ exabyte=approximately 1 billion GB) of health data by 2020 [2]. This wealth of data has been touted as a practically inexhaustible source of knowledge to fuel a learning health care system [3]. Yet at this time, significant challenges remain for using clinical data for research and optimization of health care delivery [4]. Integral to addressing these challenges and studying an intervention in actual clinical care is the ability to accurately and reliably identify patients with particular diagnoses or medical conditions across heterogeneous systems [4-6]. An EHR-based computable phenotype aims to do precisely that. Henceforth, it is referred to as an EHR-based phenotype, defined as a set of data elements and logical expressions used to identify individuals or populations (ie, cohorts) with particular diagnoses or medical conditions via clinical characteristics, events, and service patterns that are ascertained using a computerized query of an EHR system or data repository [5,7]. Phenotypes are typically used in clinical trial recruitment to identify cohorts with specific conditions using diverse data sources [5]. They are also increasingly used to define an authoritative standard for electronic clinical quality measure reporting [8].

An estimated 2.1 million people in the United States have opioid use disorder (OUD) [9], and over 33,000 opioid-related deaths occur annually, a number projected to increase to more than 81,000 by 2025 [10,11]. From 2016 to 2017, emergency departments (EDs) experienced a 30\% increase in visits for opioid overdose [12]. Buprenorphine, a partial opioid agonist generally combined with an antagonist (naloxone), is an effective treatment for OUD that decreases mortality (from approximately $5 \%$ to $3 \%$ annually following an ED visit for opioid overdose), withdrawal symptoms, craving, and opioid use [13-15]. Initiating buprenorphine in the ED doubles the rate of addiction treatment engagement in ED patients with OUD [16]. However, ED-initiated buprenorphine has not yet been adopted into routine emergency care $[17,18]$.

\section{Objectives}

Phenotyping could be used as a clinical tool to identify patients likely to benefit from ED-initiated buprenorphine or other interventions and as a research tool to identify patients who should be included in large-scale intervention studies of OUD interventions. We will conduct a multi-system pragmatic trial of user-centered clinical decision support to implement EMergency department-initiated BuprenorphinE for opioid use Disorder (EMBED) across 20 EDs in 5 health care systems [19]. EHR phenotyping will allow pragmatic comparison of the effectiveness of the EMBED intervention to usual care on outcomes in ED patients with OUD in the upcoming EMBED trial (primary outcome-adoption of ED-initiated buprenorphine in routine emergency care). Our objective in this study was to derive and validate an EHR-based computable phenotype to identify ED patients with OUD using structured data; physician validation based on chart review was used as the reference standard. This phenotype will be used to inform patient identification and data collection for the subsequent EMBED pragmatic trial.

\section{Methods}

\section{Study Setting and Sample}

This phenotype was created for the purposes of identifying patients with OUD who could benefit from ED-initiation of buprenorphine in a subsequent trial or quality improvement initiatives. Therefore, the phenotype only included ED patients who were discharged from the hospital (ie, not admitted as inpatients), were not currently prescribed buprenorphine, methadone, or naltrexone as medication treatment for OUD, and were not pregnant (as buprenorphine with naloxone may not be safe for pregnant women and its use requires more expertise than clinical decision support). This study was performed within the XXXX Health System in YYYY and XXXX Health System in YYYY by identifying a cohort of adults ( $>18$ years of age) with ED encounters between November 1, 2017, and October 31, 2018, in the EHR. The 2 health care systems use different billing companies, but the same EHR vendor (Epic; Epic Systems Corporation). Data were extracted from the EHR of each hospital using local Epic Clarity databases (Epic; Epic Systems Corporation). These data comprised information available within the EHR on the date of service of the ED visit in question. Approval for this study was provided by the Institutional Review Boards of the respective institutions (Protocol IDs 2000022749 [internal validation] and 18-2653 [external validation]).

\section{Clinical Definition of Opioid Use Disorder}

Although psychiatric evaluation is the gold standard for diagnosing OUD, within the emergency medicine (EM) context, diagnosis if performed is based on the Diagnostic and Statistical Manual of Mental Disorders, 5th Edition (DSM-5) criteria [20]. The DSM-5 specifies 11 criteria for the diagnosis of OUD, with qualifiers for remission [21]. It specifies that OUD consists of "A problematic pattern of opioid use leading to clinically significant impairment or distress, as manifested by at least two of [...eleven criteria], occurring within a 12-month period." These criteria include opioids taken repeatedly, continuously, and in larger amounts over a longer period than was intended, resulting in sequelae such as tolerance, withdrawal, craving, desire to cut down, failure to fulfill or engage in social and role obligations (such as at work, school, or home), and continued use despite problems related to use. 


\section{Electronic Health Record Definition}

The computable phenotype algorithm was developed based on data elements from available primary care OUD phenotypes [22,23] with additions and revisions based on the clinical judgment of an EM attending physician and clinical informaticist as well as available and high-yield structured ED data elements as judged by the health system's medical director of Information
Technology (HP). To maximize the yield and performance of the phenotype, 2 separate algorithms were created (Figure 1). Algorithm 1 is a diagnostic coding-based approach to identifying patients with OUD, utilizing opioid-related International Classification of Diseases, Tenth Revision, Clinical Modification (ICD-10) diagnostic codes associated with the ED visit (as coded by a clinician or medical coder, Table 1).

Figure 1. Flow diagram of phenotypes. ED: emergency department; MOUD: medication for opioid use disorder; ETOH: ethyl alcohol; OUD: opioid use disorder.

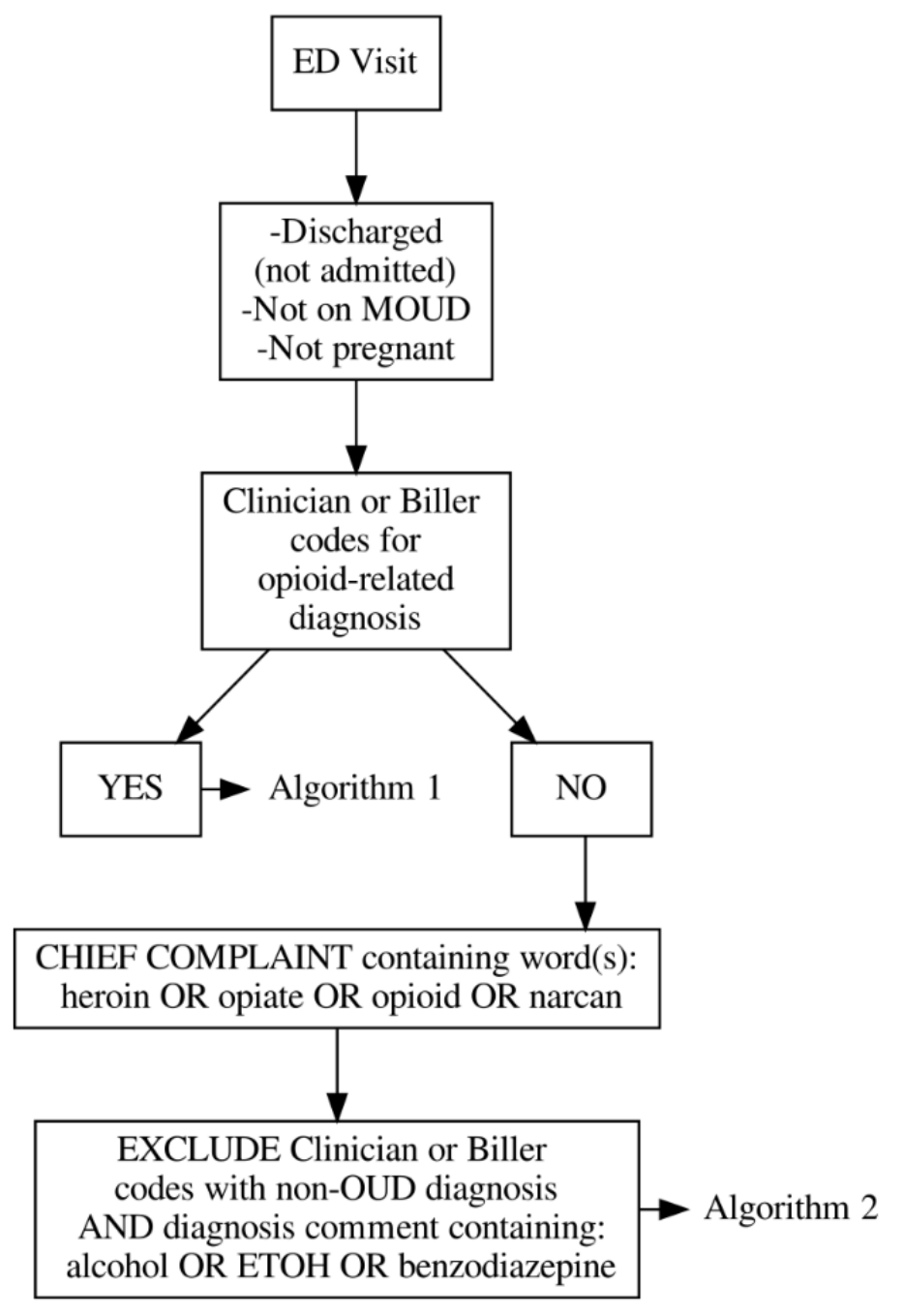

Table 1. List of International Classification of Diseases, Tenth Revision (ICD-10) codes for opioid-related diagnoses used for Algorithm 1 case detection.

\begin{tabular}{ll}
\hline ICD-10 ${ }^{\text {a code }}$ & Description \\
\hline F11 & Opioid-related disorders \\
T40.0 & Poisoning by, adverse effect of, and underdosing of opium \\
T40.1 & Poisoning by, adverse effect of, and underdosing of heroin \\
T40.2 & Poisoning by, adverse effect of, and underdosing of other opioids \\
T40.3 & Poisoning by, adverse effect of, and underdosing of methadone \\
T40.4 & Poisoning by, adverse effect of, and underdosing of other synthetic narcotics \\
T40.6 & Poisoning by, adverse effect of, and underdosing of other and unspecified narcotics \\
\hline
\end{tabular}

${ }^{a}$ ICD-10: International Classification of Diseases, Tenth Revision. 
Algorithm 2 identifies patients who have not been captured by Algorithm 1 but have information in their ED chief complaint suggestive of OUD. This algorithm flagged patients if the words heroin, opiate, opioid, or narcan were included in their chief complaint for the ED visit. However, as naloxone is often used in ED patients with undifferentiated altered mental status or overdose, patients with narcan in their chief complain who did not have an OUD-related final diagnosis were excluded. Upon preliminary testing of the algorithm, the 2 most frequent false-positive diagnoses were alcohol- and benzodiazepine-related. Visits with these chief complaints but alcohol- and benzodiazepine-related final diagnoses were removed by excluding patients with the words alcohol or benzodiazepine in their final ED diagnosis.

\section{Structured Query Language Implementation of the Phenotype}

After the 2 algorithms were reviewed, finalized, and approved by the investigative team, individual elements of each algorithm were converted into structured query language (SQL). The computable phenotype algorithm was written to be deployed in the Epic EHR systems across both the health systems. Data structures within Epic were mapped to each of the concepts (which were standardized across hospitals in the system) by a clinical informatics expert (HP). This eliminated the necessity of translating concepts to local codes within hospitals. Sample queries were run, and HP verified charts for accuracy. The SQL query and data dictionary (Multimedia Appendix 1, SQL file and data dictionary) were assembled by HP and reviewed for accuracy and comprehensiveness by HP, EM, and DC. The possible values of each variable are described in Figure 1 and expanded upon in Tables 1 and 2. Once all of the elements of the phenotype were codified in SQL, the algorithms were applied to the study population's ED medical records.

Table 2. Algorithm 2 case definition variables.

\begin{tabular}{ll}
\hline EHR $^{\mathrm{a}}$ data variable & Criteria for suspected opioid use disorder \\
\hline Chief complaint & - $\quad$ Reason for visit contains the words heroin; opiate; opioid \\
& $-\quad$ Reason for visit comment contains the word narcan \\
Diagnosis description & - Not Algorithm 1 positive, that is, does not contain ICD-10 codes F11 or T40. 0-40.6 listed in Table 1 \\
& $-\quad$ Does not contain the words alcohol, EtOH, benzodiazepine
\end{tabular}

${ }^{\mathrm{a} E H R}$ : electronic health record.

${ }^{\mathrm{b}}$ ICD-10: International Classification of Diseases, Tenth Revision.

\section{Evaluation Phase 1: Internal Validation}

Following the implementation of the computable phenotype algorithm, internal validation was performed using a sample of 125 charts retrieved from the XXXX health system EHR by a clinical informaticist (HP). A total of 75 charts were intended to be representative of the resulting OUD phenotypes with 50 of these charts meeting Algorithm 1 criteria and the other 25 meeting Algorithm 2 criteria. The other 50 charts were phenotype negative (ie, not satisfying criteria for either algorithm). Charts were selected at random from the cohort with ED visits from April 10, 2018, to August 1, 2018, across the health systems and reviewed during August 2018 to October 2018 for the internal validation phase and December 2018 to January 2019 for the external validation phase. As the chart reviewers were given access to the patient's full chart, the time window for the charts was deliberately narrow to avoid postvisit information (eg, of someone who subsequently develops OUD that was not present on the date of the ED visit) confounding the accuracy of the chart review of the ED visit.

\section{Evaluation Phase 2: External Validation}

The external validation cohort was constructed by a clinical informaticist (WKR) with 20,000 randomly sampled ED visits occurring between November 1, 2017, and October 31, 2018 across the XXXXX health system. We picked this number of charts given the rate of phenotype-positive charts in the internal validation cohort with a goal of estimating sensitivity of the phenotype based on prevalence in this random sample. A total of 55 charts met Algorithm 1 criteria. Of those not positive for Algorithm 1, 1 chart met Algorithm 2 criteria. Of the remaining negative cases, a $0.25 \%$ (50/200) random sample produced 50 charts for review. Cases positive for Algorithm 1 or 2 were combined owing to the low yield of a single chart identified as Algorithm 2 positive.

\section{Chart Review}

Each chart was reviewed independently and separately by 2 EM physicians (internal validation: DPN, EB; external validation: $\mathrm{CH}, \mathrm{AMS}$ ) blinded to the results and the algorithms and the decision of the other reviewer. All cases of disagreement were adjudicated by a third EM physician reviewer (internal validation: $\mathrm{KC}$; external validation: TFP) also blinded to the results of the algorithms and the decision of the other reviewers. Reviewers were asked to diagnose patients as OUD-positive or OUD-negative based upon a review of EHR data available up to and on the date of the ED visit (but not after the ED visit), their clinical judgment, and the DSM-5 OUD diagnostic criteria which were presented to them with each case at the time of review [21]. For cases that were categorized as OUD-positive, reviewers were then prompted to select at least 2 of the 11 DSM-5 criteria that informed their diagnosis.

\section{Analysis}

Phenotype performance was assessed using descriptive statistics. A standard $2 \times 2$ confusion matrix [24] was configured for 
analysis of the performance of each algorithm in each phase. The reference standard was the adjudicated diagnosis, whereas the test was the phenotype result. For Algorithm 1 in the internal validation phase (Table 3), the top row included the 50 phenotype-positive charts, and the bottom row included the 50 phenotype-negative charts. For Algorithm 2 in the internal validation phase (Table 3), the top row included the 25 phenotype-positive charts, and the bottom row included the 25 phenotype-negative charts. In the external validation phase, the algorithms were combined because of low incidence of Algorithm 2-positive (Table 3), with 56 positive and 50 negative. Interrater reliability was reported using Cohen kappa. Analyses were conducted with the scikit-learn package (version 0.19.2) in Python (version 2.7.12) for internal validation and Stata (StataCorp, version 14) for external validation.

Table 3. Confusion matrices for validation phases (disease present: reference standard).

\begin{tabular}{|c|c|c|c|c|}
\hline \multirow[t]{2}{*}{ Test } & \multicolumn{4}{|l|}{ Result } \\
\hline & Reviewers + & Reviewers - & Predictive value & $95 \% \mathrm{CI}$ \\
\hline \multicolumn{5}{|c|}{ Algorithm 1 (internal validation) } \\
\hline Phenotype + & 48 & 2 & $0.96^{\mathrm{a}}$ & $0.863-0.995$ \\
\hline Phenotype - & 1 & 49 & $0.98^{\mathrm{b}}$ & 0.893-0.999 \\
\hline \multicolumn{5}{|c|}{ Algorithm 2 (internal validation) } \\
\hline Phenotype + & 20 & 5 & $0.8^{\mathrm{a}}$ & $0.593-0.932$ \\
\hline Phenotype - & 0 & 25 & $1.0^{\mathrm{b}}$ & $0.863-1.000^{\mathrm{c}}$ \\
\hline \multicolumn{5}{|c|}{ Combined phenotype (external validation) } \\
\hline Phenotype + & 53 & 3 & $0.95^{\mathrm{a}}$ & $0.851-0.989$ \\
\hline Phenotype - & 4 & 46 & $0.92^{\mathrm{b}}$ & $0.807-0.978$ \\
\hline
\end{tabular}

${ }^{\text {a}}$ Positive predictive value.

$\mathrm{b}_{\text {Negative predictive value. }}$

${ }^{\mathrm{c}} 97.5 \%$, one-sided.

\section{Results}

Among ED visits resulting in discharge from November 1, 2017, to October 31, 2018, across the 13 EDs in the 2 health care systems, a total of 474,176 unique ED visits (discharged patients only) with an average of $36,475 \mathrm{ED}$ visits per year per site were identified. A total of 2294 of these visits were phenotype-positive with an average of 176 (median 104) phenotype-positive visits per site. Site visit by volume is presented in Table 4. 
Table 4. Annual volume of emergency department (ED) visits meeting phenotype criteria (November 1, 2017, to October 31, 2018, ED discharges only).

\begin{tabular}{|c|c|c|c|c|}
\hline Validation & Total patients (n) & Total visits (n) & Algorithm 1 (n) & Algorithm 2 (n) \\
\hline \multicolumn{5}{|l|}{ Internal } \\
\hline \multicolumn{5}{|l|}{ Department } \\
\hline Hospital X I & 44,291 & 67,995 & 343 & 49 \\
\hline Health System X II & 22,344 & 29,309 & 56 & 11 \\
\hline Health System X III & 24,738 & 38,128 & 251 & 46 \\
\hline Health System X IV & 27,220 & 44,505 & 324 & 73 \\
\hline Health System X V & 44,780 & 65,837 & 509 & 70 \\
\hline Health System X VI & 17,797 & 22,540 & 25 & 0 \\
\hline Total & 181,170 & 268,314 & 1508 & 249 \\
\hline Average & 30,195 & 44,719 & 251.3 & 41.5 \\
\hline \multicolumn{5}{|l|}{ External } \\
\hline \multicolumn{5}{|l|}{ Department } \\
\hline Health System Y I & 9818 & 15,749 & 37 & 4 \\
\hline Health System Y II & 15,220 & 25,556 & 91 & 2 \\
\hline Health System Y III & 22,332 & 30,912 & 57 & 4 \\
\hline Health System Y IV & 22,080 & 38,086 & 100 & 4 \\
\hline Health System Y V & 5467 & 6190 & 24 & 1 \\
\hline Health System Y VI & 34,576 & 46,335 & 98 & 0 \\
\hline Health System Y VII & 32,879 & 43,034 & 110 & 5 \\
\hline Total & 142,372 & 205,862 & 517 & 20 \\
\hline Average & 20,339 & 29,409 & 74 & 3 \\
\hline
\end{tabular}

\section{Internal Validation Cohort}

In the internal validation cohort of 125 charts, reviewers disagreed on the classification of 3 charts (agreement $=97 \%$; kappa $=0.95$ ), with the adjudicator identifying the 2 discordant Algorithm 1 cases as not having OUD and the 1 discordant Algorithm 2 case as having OUD. Algorithm 1 had a positive predictive value (PPV) of 0.96 (95\% CI 0.863-0.995) and a negative predictive value (NPV) of 0.98 (95\% CI 0.893-0.999; Table 3). Algorithm 2 had a PPV of 0.8 (95\% CI 0.593-0.932) and an NPV of 1.0 (one-sided 97.5\% CI 0.863-1; Table 3). The most frequently met current DSM- 5 criteria were "opioids taken in larger amounts or over a longer period than was intended" or "recurrent use in situations in which it is physically hazardous," whereas the least frequent criteria were those describing social dysfunction related to the use of opioids (such as "recurrent opioid use resulting in a failure to fulfill major role obligations at work, school, or home" or "important social, occupational, or recreational activities are given up or reduced because of opioid use").

\section{External Validation Cohort}

In the external validation cohort of 106 charts, reviewers disagreed on the classification of 8 charts (agreement $=92.5 \%$; kappa $=0.85$ ). A total of 3 of the 8 discordant cases were phenotype-positive, of which the adjudicator determined 2 as having OUD. Of the 5 discordant cases that were phenotype-negative, the adjudicator identified 3 as having OUD. The combined phenotype had a PPV of 0.95 (95\% CI 0.851-0.989) and an NPV of 0.92 (95\% CI 0.807-0.978; Table $3)$. The most frequently met current DSM-5 criteria were "opioids are often taken in larger amounts or over a longer period than was intended" and "craving, or a strong desire or urge to use opioids," whereas the least frequent criterion was "important social, occupational, or recreational activities are given up or reduced because of opioid use."

\section{Discussion}

\section{Principal Findings}

With an externally validated PPV of 0.95 and NPV of 0.92 , the combined phenotype derived and validated for this study performed remarkably well in predicting OUD in ED patients across 2 large health care systems. The strength of the phenotype's classification performance may be because of the possibility that the algorithm and the reviewers were using similar (if not the same) information from patients' charts.

In both the internal and external validation chart reviews, the most common DSM criterion selected by the reviewers was "opioids are often taken in larger amounts or over a longer period than was intended." In the internal validation phase, the second most common criterion was "recurrent opioid use in situations in which it is physically hazardous," whereas the 
second most common criterion in the external validation phase was "craving, or a strong desire or urge to use opioids." Although desire and effort to cut down or control opioid use are specific diagnostic criteria, they were inconsistently applied by the reviewers. As these specific criteria are not explicitly documented in the routine emergency care, the reviewers instead had to infer which criteria to apply to cases using available documentation. In both chart review phases, the least frequently identified criteria were those describing failures in social behavior as they pertained to the use of opioids. This could be because of the fact that ED billing requirements do not require detailed documentation of social history, and the impact of opioids on social behaviors usually has limited value for assisting clinicians in making a diagnosis during emergency care [25].

Given the limitations of ED documentation, our phenotype benefited from incorporation of available structured data elements from the data dictionaries created in previous work to develop EHR phenotypes for primary care patients on chronic opioid therapy at risk for problematic opioid use [22,23]. Given the difference in populations and objectives between this study and the primary care OUD phenotype, it is difficult to compare the differences in their phenotypes' performance. In particular, the previous work focused on the performance of natural language processing for identifying risk for problematic opioid use in patients for whom differences in the signs and symptoms of OUD might be more nuanced: every patient included in that study was on chronic opioid therapy. The goal of that study was to capture the presence of OUD symptoms using free-text notes-a complex machine learning problem. In contrast, our study included a broader population (all patients presenting to the ED were eligible for inclusion in the phenotype-negative sample), and our phenotype drew on structured data elements including diagnoses and chief complaints; these structured data generally reflect the clinical judgment of people who have directly observed the patient and determined that OUD was likely.

A strength of our study compared with previous EHR phenotype work is the external validation of the phenotype's performance via chart review in the second health care system. For example, the HIV EHR phenotype developed by Paul et al [26] performs well, but its transportability and performance in outside health care systems are not known [27]. External validation is particularly important for EHR phenotypes that rely on documentation and diagnostic codes as documentation and diagnostic codes are dependent on local practice patterns by clinicians and coders, both of which could vary within and across health care systems.

The phenotype described here will be used as part of a subsequent pragmatic trial to be deployed across multiple health care systems to identify patients who may have been candidates for ED-initiated buprenorphine-these cases will form the denominator of a measure to assess what proportion of those potentially eligible actually received buprenorphine. As most patients evaluated using the phenotype will screen negative, our approach would likely result in a high number of false negatives in a true epidemiologic evaluation. As we were trying to maximize specificity for a pragmatic trial, the phenotype's classification performance will meet the trial's needs to screen patients for eligibility for ED-initiated buprenorphine with high specificity. Furthermore, the goal was not to definitively determine a diagnosis of OUD for each patient. For clinical practice, any patient identified as having OUD by this phenotype would require confirmation using an in-person assessment. As the capacity and expectation of EDs to treat OUD expands, so also does the value of an accurate EHR phenotype that could be used to identify patients who might benefit from treatment including ED-initiated buprenorphine and referral for ongoing medication treatment for OUD.

\section{Limitations}

The primary limitation of this study is the use of retrospective ED chart review as a reference standard for the diagnosis of OUD. Our chart review process was robust and included all clinical documentation up to date of the ED visit. However, a full diagnostic assessment by a psychiatrist or addiction medicine specialist would be the gold standard to establish a diagnosis of OUD. If available, it is possible that such an assessment would differ from chart review alone.

External validation in an outside health care system strengthens the evidence for the generalizability of our phenotype. The external system uses the same EHR vendor but a different billing and coding company. It is unknown how the EHR phenotype would perform in systems using other EHR platforms. In addition, transportability issues have been discovered in preliminary estimates from a third health system because of differences in structured data capture of the chief complaint. In the external validation phase, the second algorithm did not identify a substantial number of cases. This suggests that there are likely local practice patterns in documentation or coding that may have affected the transportability of this EHR phenotype [27]. Although local phenotype development and adaptation could overcome this limitation, the overall classification performance remained strong in the external validation phase. Furthermore, in the internal validation phase, the individual algorithms maintained high PPV values (0.96 and 0.8, respectively) and NPV values (0.98 and 1.0).

In future work, the efficacy of the phenotype algorithms will be tested in the EMBED trial, and the question as to whether these algorithms can function in a pragmatic ED setting will be answered. Statistically, the cohort selection and chart review performed here did not obtain a patient population reflective of the true prevalence of the disease, and as such, sensitivity and specificity calculations would not provide an accurate reflection of the phenotype's performance in a true ED population. Assessment of sensitivity for events with low base rates is inherently unreliable. A very large sample size would be necessary for a precise estimate of sensitivity. Therefore, the assessment of sensitivity in this analysis is limited. To address this limitation, we report only PPV and NPV here. Estimating sensitivity and specificity for the external validation study can be done by inflating the phenotype-negative row numbers in the external validation confusion matrix by the sampling factor (Table 3) to represent 19,944 patients who screened negative. The sampling factor of approximately $399(19,944 / 50)$ would change this row to extrapolated values of 1596 (false negatives) 
and 18,354 (true negatives). These extrapolated values would yield a sensitivity of $3.2 \%$ with an extremely wide confidence interval and specificity of $99.9 \%$. This wide range could be explained by the false negatives that the phenotype is not identifying. Given the current opioid crisis, we know that the rates of OUD are high, and there are likely many individuals with occult OUD that is not being identified in the ED as they may be presenting with medical complaints unrelated to their OUD comorbidity. For example, abdominal pain and chest pain are the 2 most common presenting complaints to EDs nationally. There is likely a large population of ED patients with these complaints that have OUD that goes unrecognized in the ED. Future work should screen for more precise estimates of undiagnosed OUD in the ED population.

In the upcoming trial, further evaluation of the phenotype algorithms' performance will begin to address the intra- and intersite population sensitivity and specificity. To further refine the algorithm's ability to discern between true and false positives, logistic regression is planned to predict future OUD-coded diagnoses given information from previous visits, such that variables can be removed given their performance in the regression model. Future work will also attempt to quantify the rate of false negatives through extended manual review and to determine whether changes to the algorithm improve sensitivity. The long-term goal of future work is to standardize the representation of the algorithms such that they can be portable beyond Epic to other EHR vendors as well as explore additional information retrieval techniques [28]. A more comprehensive validation could establish more reliable sensitivity estimates by use of a gold standard estimate of true prevalence of OUD in the ED population by screening a representative ED population for OUD with DSM-5 diagnostic criteria [21].

\section{Conclusions}

An EHR phenotype derived and internally and externally validated for the purposes of a pragmatic trial to test the effectiveness of user-centered clinical decision support to increase the adoption of ED-initiated buprenorphine performed reliably and accurately to identify ED patients with OUD. The 2 algorithms comprising the phenotype were transportable across health care systems and have potential value for both clinical quality improvement interventions as well as research endeavors. Standardization of the phenotype will support efforts to use clinical phenotyping as an evidence-based tool at the front line of clinical practice.

\section{Acknowledgments}

Research reported in this publication was supported within the National Institutes of Health (NIH) Health Care Systems Research Collaboratory, by a cooperative agreement (UG3DA047003) from the National Institute on Drug Abuse of the NIH. This work also received logistical and technical support from the NIH Collaboratory Coordinating Center (U24AT009676). The content is solely the responsibility of the authors and does not necessarily represent the official views of the NIH. The funders had no role in study design, data collection, and analysis, decision to publish, or preparation of the manuscript.

\section{Authors' Contributions}

DC, ERM, HP, TFP, and JDD significantly contributed to the conception and design of the study. HP, WKR, TFP, MP, and DC acquired and analyzed the data. DPN, EB, KCC, CH, AMS, and TFP performed the clinical chart reviews. HP, DC, DPN, and ERM drafted the initial manuscript. All authors were involved in data interpretation, revised the manuscript, and approved the final version submitted for publication. HP, DC, WKR, and TFP had access to the data in the study and took responsibility for data integrity and accuracy. ERM took responsibility for all aspects of the work.

\section{Conflicts of Interest}

None declared.

\section{Multimedia Appendix 1}

SQL code to retrieve records from Epic Clarity database.

[TXT File, 71 KB-Multimedia Appendix 1]

\section{References}

1. Henry J, Pylypchuk Y, Searcy T, Patel V. Health IT Dashboard. 2016. Adoption of Electronic Health Record Systems among US Non-Federal Acute Care Hospitals: 2008-2015 URL: https://dashboard.healthit.gov/evaluations/data-briefs/ non-federal-acute-care-hospital-ehr-adoption-2008-2015.php [accessed 2019-10-15]

2. Cyclone Interactive: Digital Marketing Agency. 2014. Vertical Industry Brief: Digital Universe Driving Data Growth in Healthcare Internet URL: https://www.cycloneinteractive.com/cyclone/assets/File/ digital-universe-healthcare-vertical-report-ar.pdf [accessed 2015-12-29]

3. Krumholz HM. Big data and new knowledge in medicine: the thinking, training, and tools needed for a learning health system. Health Aff (Millwood) 2014 Jul;33(7):1163-1170 [FREE Full text] [doi: 10.1377/hlthaff.2014.0053] [Medline: 25006142] 
4. Richesson RL, Green BB, Laws R, Puro J, Kahn MG, Bauck A, et al. Pragmatic (trial) informatics: a perspective from the NIH Health Care Systems Research Collaboratory. J Am Med Inform Assoc 2017 Sep 1;24(5):996-1001. [doi: 10.1093/jamia/ocx016] [Medline: 28340241]

5. Shivade C, Raghavan P, Fosler-Lussier E, Embi PJ, Elhadad N, Johnson SB, et al. A review of approaches to identifying patient phenotype cohorts using electronic health records. J Am Med Inform Assoc 2014;21(2):221-230 [FREE Full text] [doi: 10.1136/amiajnl-2013-001935] [Medline: 24201027]

6. Loudon K, Treweek S, Sullivan F, Donnan P, Thorpe KE, Zwarenstein M. The PRECIS-2 tool: designing trials that are fit for purpose. Br Med J 2015 May 8;350:h2147. [doi: 10.1136/bmj.h2147] [Medline: 25956159]

7. Richesson RL, Hammond WE, Nahm M, Wixted D, Simon GE, Robinson JG, et al. Electronic health records based phenotyping in next-generation clinical trials: a perspective from the NIH Health Care Systems Collaboratory. J Am Med Inform Assoc 2013 Dec;20(e2):e226-e231 [FREE Full text] [doi: 10.1136/amiajnl-2013-001926] [Medline: 23956018]

8. Bodenreider O, Nguyen D, Chiang P, Chuang P, Madden M, Winnenburg R, et al. The NLM value set authority center. Stud Health Technol Inform 2013;192:1224 [FREE Full text] [Medline: 23920998]

9. Ahrnsbrak R, Bose J, Hedden S, Lipari R, Park-Lee E. SAMHSA - Substance Abuse and Mental Health Services. 2017. Key Substance Use and Mental Health Indicators in the United States: Results from the 2016 National Survey on Drug Use and Health URL: https://www.samhsa.gov/data/sites/default/files/NSDUH-FFR1-2016/NSDUH-FFR1-2016.htm [accessed 2017-09-01]

10. Seth P, Scholl L, Rudd RA, Bacon S. Overdose deaths involving opioids, cocaine, and psychostimulants - United States, 2015-2016. MMWR Morb Mortal Wkly Rep 2018 Mar 30;67(12):349-358 [FREE Full text] [doi: 10.15585/mmwr.mm6712a1] [Medline: 29596405]

11. Chen Q, Larochelle MR, Weaver DT, Lietz AP, Mueller PP, Mercaldo S, et al. Prevention of prescription opioid misuse and projected overdose deaths in the United States. JAMA Netw Open 2019 Feb 1;2(2):e187621 [FREE Full text] [doi: 10.1001/jamanetworkopen.2018.7621] [Medline: $\underline{30707224]}$

12. Vivolo-Kantor AM, Seth P, Gladden RM, Mattson CL, Baldwin GT, Kite-Powell A, et al. Vital signs: trends in emergency department visits for suspected opioid overdoses - United States, July 2016-September 2017. MMWR Morb Mortal Wkly Rep 2018 Mar 9;67(9):279-285 [FREE Full text] [doi: 10.15585/mmwr.mm6709e1] [Medline: 29518069]

13. Kakko J, Svanborg KD, Kreek MJ, Heilig M. 1-year retention and social function after buprenorphine-assisted relapse prevention treatment for heroin dependence in Sweden: a randomised, placebo-controlled trial. Lancet 2003 Feb 22;361(9358):662-668. [doi: 10.1016/S0140-6736(03)12600-1] [Medline: 12606177]

14. Mattick RP, Breen C, Kimber J, Davoli M. Buprenorphine maintenance versus placebo or methadone maintenance for opioid dependence. Cochrane Database Syst Rev 2014 Feb 6(2):CD002207. [doi: 10.1002/14651858.CD002207.pub4] [Medline: 24500948]

15. Larochelle MR, Bernson D, Land T, Stopka TJ, Wang N, Xuan Z, et al. Medication for opioid use disorder after nonfatal opioid overdose and association with mortality: a cohort study. Ann Intern Med 2018 Aug 7;169(3):137-145 [FREE Full text] [doi: 10.7326/M17-3107] [Medline: 29913516]

16. D'Onofrio G, O'Connor P, Pantalon M, Chawarski M, Busch S, Owens P, et al. Emergency department-initiated buprenorphine/naloxone treatment for opioid dependence: a randomized clinical trial. J Am Med Assoc 2015 Apr 28;313(16):1636-1644 [FREE Full text] [doi: 10.1001/jama.2015.3474] [Medline: 25919527]

17. Duber HC, Barata IA, Cioè-Peña E, Liang SY, Ketcham E, Macias-Konstantopoulos W, et al. Identification, management, and transition of care for patients with opioid use disorder in the emergency department. Ann Emerg Med 2018 Oct;72(4):420-431 [FREE Full text] [doi: 10.1016/j.annemergmed.2018.04.007] [Medline: 29880438]

18. Martin A, Mitchell A, Wakeman S, White B, Raja A. Emergency department treatment of opioid addiction: an opportunity to lead. Acad Emerg Med 2018 May;25(5):601-604 [FREE Full text] [doi: 10.1111/acem.13367] [Medline: 29266577]

19. NIH Collaboratory Rethinking Clinical Trials. UG3 Project: Pragmatic Trial of User-Centered Clinical Decision Support to Implement EMergency Department-Initiated BuprenorphinE for Opioid Use Disorder (EMBED) - Rethinking Clinical Trials Internet URL: https://tinyurl.com/y35dz7b8 [accessed 2018-10-25]

20. Bernstein E, Bernstein J, Weiner S, D'Onofrio G. Substance use disorders. In: Tintinalli JE, Stapczynski JS, Ma OJ, Yealy DM, Meckler GD, Cline DM, editors. Tintinalli's Emergency Medicine: A Comprehensive Study Guide. Eight Edition. New York: McGraw-Hill; 2016.

21. American Psychiatric Association. Substance-related addictive disorders. In: Diagnostic and Statistical Manual of Mental Disorders. Arlington, VA: American Psychiatric Association; 2013.

22. Carrell DS, Cronkite D, Palmer RE, Saunders K, Gross DE, Masters ET, et al. Using natural language processing to identify problem usage of prescription opioids. Int J Med Inform 2015 Dec;84(12):1057-1064. [doi: 10.1016/j.ijmedinf.2015.09.002] [Medline: 26456569]

23. Palmer RE, Carrell DS, Cronkite D, Saunders K, Gross DE, Masters E, et al. The prevalence of problem opioid use in patients receiving chronic opioid therapy: computer-assisted review of electronic health record clinical notes. Pain 2015 Jul;156(7):1208-1214. [doi: 10.1097/j.pain.0000000000000145] [Medline: 25760471]

24. Hulley S, Cummings S, Browner W, Grady D, Newman T. Designing Clinical Research. Philadelphia, PA: Lippincott Williams \& Wilkins; 2013. 
25. Centers for Medicare and Medicaid Services. 1997. 1997 Documentation Guidelines for Evaluation and Management Services URL: https://www.cms.gov/Outreach-and-Education/Medicare-Learning-Network-MLN/MLNEdWebGuide/ Downloads/97Docguidelines.pdf [accessed 2019-10-09]

26. Paul DW, Neely NB, Clement M, Riley I, Al-Hegelan M, Phelan M, et al. Development and validation of an electronic medical record (EMR)-based computed phenotype of HIV-1 infection. J Am Med Inform Assoc 2018 Feb 1;25(2):150-157 [FREE Full text] [doi: 10.1093/jamia/ocx061] [Medline: 28645207]

27. Pacheco JA, Rasmussen LV, Kiefer RC, Campion TR, Speltz P, Carroll RJ, et al. A case study evaluating the portability of an executable computable phenotype algorithm across multiple institutions and electronic health record environments. J Am Med Inform Assoc 2018 Nov 1;25(11):1540-1546 [FREE Full text] [doi: 10.1093/jamia/ocy101] [Medline: 30124903 ]

28. Rasmussen LV, Thompson WK, Pacheco JA, Kho AN, Carrell DS, Pathak J, et al. Design patterns for the development of electronic health record-driven phenotype extraction algorithms. J Biomed Inform 2014 Oct;51:280-286 [FREE Full text] [doi: 10.1016/j.jbi.2014.06.007] [Medline: 24960203]

\author{
Abbreviations \\ DSM-5: Diagnostic and Statistical Manual of Mental Disorders, 5th Edition \\ ED: emergency department \\ EHR: electronic health record \\ EM: emergency medicine \\ EMBED: EMergency department-initiated BuprenorphinE for opioid use Disorder \\ ICD-10: International Classification of Diseases, Tenth Revision \\ NIH: National Institutes of Health \\ NPV: negative predictive value \\ OUD: opioid use disorder \\ PPV: positive predictive value \\ SQL: structured query language
}

Edited by G Eysenbach; submitted 08.08.19; peer-reviewed by X Fan, M Graber, R Radecki, C Freiermuth, E Schoenfeld, JT Pollettini,
A Follmann, KA Nguyen; comments to author 18.09.19; revised version received 27.09.19; accepted 01.10.19; published 31.10.19
Please cite as:
Chartash D, Paek H, Dziura JD, Ross BK, Nogee DP, Boccio E, Hines C, Schott AM, Jeffery MM, Patel MD, Platts-Mills TF, Ahmed
O, Brandt C, Couturier K, Melnick E
Identifying Opioid Use Disorder in the Emergency Department: Multi-System Electronic Health Record-Based Computable Phenotype
Derivation and Validation Study
JMIR Med Inform 2019;7(4):e15794
URL: http://medinform.jmir.org/2019/4/e15794/
doi: $10.2196 / 15794$
PMID: $\underline{31674913}$

CDavid Chartash, Hyung Paek, James D Dziura, Bill K Ross, Daniel P Nogee, Eric Boccio, Cory Hines, Aaron M Schott, Molly M Jeffery, Mehul D Patel, Timothy F Platts-Mills, Osama Ahmed, Cynthia Brandt, Katherine Couturier, Edward Melnick. Originally published in JMIR Medical Informatics (http://medinform.jmir.org), 31.10.2019. This is an open-access article distributed under the terms of the Creative Commons Attribution License (https://creativecommons.org/licenses/by/4.0/), which permits unrestricted use, distribution, and reproduction in any medium, provided the original work, first published in JMIR Medical Informatics, is properly cited. The complete bibliographic information, a link to the original publication on http://medinform.jmir.org/, as well as this copyright and license information must be included. 